FERMILAB-PUB-92/264-T

LBL-32987

hep-ph@xxx/yymmnn

\title{
Flavor Asymmetry of the Nucleon Sea: Consequences for Dilepton Production
}

\author{
Estia J. Eichten* \\ Fermi National Accelerator Laboratory P.O. Box 500, Batavia, Illinois 60510 \\ Ian Hinchliffel \\ Lawrence Berkeley Laboratory Berkeley, California 94720 \\ Chris Quigg或 \\ Fermi National Accelerator Laboratory P.O. Box 500, Batavia, Illinois 60510
}

\begin{abstract}
Parton distributions derived from a chiral quark model that generates an excess of down quarks and antiquarks in the proton's sea satisfactorily describe the measured yields of muon pairs produced in proton-nucleus collisions. Comparison of dilepton yields from hydrogen and deuterium targets promises greater sensitivity to the predicted flavor asymmetry.
\end{abstract}

Typeset Using REVTEX

\footnotetext{
*Internet address: eichten@fnal.fnal.gov.

${ }^{\dagger}$ Internet address: hinchliffe\%theorm.hepnet@lbl.gov.

${ }^{\ddagger}$ Internet address: quigg@fnal.fnal.gov.
} 
In a recent article [1], we showed that the fluctuation of constituent quarks into quarks plus Goldstone bosons generates a flavor-asymmetry in the light-quark sea of the nucleon that is a plausible origin for the violation of the Gottfried sum rule reported by the New Muon Collaboration (NMC) at CERN [2]. Fermilab experiment E772 has now presented measurements of the yields of massive muon pairs in collisions of $800-\mathrm{GeV} / c$ protons with nuclear targets that are sensitive to the flavor content of the nucleon sea [3]. We show in this paper that our picture of the Gottfried-sum-rule defect also accounts for the new dilepton data, and we make predictions for a more sensitive test using hydrogen and deuterium targets.

Forward (Feynman- $x_{F} \gtrsim 0.1$ ) production of massive dilepton pairs in high-energy collisions of protons with nuclear targets is dominated by the annihilation of a $u$-quark from the beam with a $\bar{u}$-antiquark from the target, and so is sensitive to the distribution of antiquarks in the target nucleons. Charge symmetry relates the distribution of up-antiquarks in the neutron to the distribution of down-antiquarks in the proton,

$$
\bar{u}^{(n)}\left(x, Q^{2}\right)=\bar{d}^{(p)}\left(x, Q^{2}\right)
$$

where $x$ is Bjorken's scaling variable and $Q^{2}$ labels the scale on which the parton distributions are measured. The yield per nucleon $\sigma_{A}$ in proton collisions with nucleus $A$ differs from the yield per nucleon $\sigma_{\text {isoscalar }}$ in proton collisions with an isoscalar target by the factor

$$
R_{A}(x) \equiv \frac{\sigma_{A}(x)}{\sigma_{\text {isoscalar }}(x)} \approx 1+\frac{(N-Z)}{A} \frac{\bar{d}(x)-\bar{u}(x)}{\bar{d}(x)+\bar{u}(x)},
$$

where $A, Z$, and $N$ are the atomic weight, atomic number, and number of neutrons in the target, and the antiquark densities refer to the proton. The approximate equality follows upon neglect of $d \bar{d}$ annihilations. The deviation of $R_{A}(x)$ from unity measures the flavorasymmetry of the light-quark sea.

The possibility that the light-quark sea contains unequal numbers of up and down quarks has been raised by the New Muon Collaboration's determination [2] of the integral

$$
I_{G}=\int_{0}^{1} d x \frac{\left[F_{2}^{\mu p}\left(x, Q^{2}\right)-F_{2}^{\mu n}\left(x, Q^{2}\right)\right]}{x}=0.240 \pm 0.016 .
$$


In the quark-parton model, the integral can be expressed as

$$
I_{G}=\frac{1}{3}+\frac{2}{3} \int_{0}^{1} d x\left[\bar{u}\left(x, Q^{2}\right)-\bar{d}\left(x, Q^{2}\right)\right] .
$$

The Gottfried sum rule [4, $I_{G}=1 / 3$, follows from the assumption that the sea is up-down symmetric. The observed defect implies a small excess,

$$
\int_{0}^{1} d x\left[\bar{u}\left(x, Q^{2}\right)-\bar{d}\left(x, Q^{2}\right)\right]=-0.14 \pm 0.024
$$

of down quarks in the sea.

Because we expect $I_{G}$ to be essentially independent of $Q^{2}$ [5], it is convenient to analyze the flavor content of the sea at a momentum scale relevant to hadron structure, where the important degrees of freedom are constituent quarks, Goldstone bosons, and gluons. Consider a proton composed of three constituent quarks: uud. An excess of down quarks over up quarks in the sea arises naturally from the isospin-respecting fluctuation of the constituent quarks into quarks and pions, the lightest of the Goldstone bosons, viz. $u \rightarrow\left(\pi^{+} d, \pi^{0} u\right)$ and $d \rightarrow\left(\pi^{0} d, \pi^{-} u\right)$. If $a$ denotes the probability for a constituent up quark to turn into a down quark and a $\pi^{+}$(containing a $u$-quark and a $\bar{d}$-antiquark), the proton composition after one iteration is $(2+7 a / 4) u+(1+11 a / 4) d+(7 a / 4) \bar{u}+(11 a / 4) \bar{d}$. The valence composition remains $u_{v}=(u-\bar{u})=2$ and $d_{v}=(d-\bar{d})=1$, but the sea contains an excess of down quarks and antiquarks over up quarks and antiquarks.

In Ref. [1], we implemented this picture in the framework of the effective chiral quark model formulated by Manohar and Georgi [6]. A straightforward calculation of the probability for a constituent quark to fluctuate leads to $I_{G}=0.278$, encouragingly close to the experimental value (31). After adjusting the ultraviolet cutoff of the chiral quark model to better reproduce the observed Gottfried-sum-rule defect, we constructed parton distributions based on the Eichten-Hinchliffe-Lane-Quigg (EHLQ) Set 1 distributions [7]. These new distributions give a good account of the NMC measurements of the Gottfried integral, the difference $F_{2}^{\mu p}-F_{2}^{\mu n}$, and the ratio $F_{2}^{\mu n} / F_{2}^{\mu p}$. Details of the construction of the parton distributions and the comparison with data may be found in Ref. [怔. For present purposes, 
it is important to note that the flavor-asymmetric sea generated by chiral field theory, which is concentrated at small values of $x$, is a small perturbation on the flavor-symmetric sea of EHLQ Set 1 [7], $x \bar{u}(x)=x \bar{d}(x)=0.182(1-x)^{8.54}$.

The production of massive muon pairs in proton-nucleus collisions offers another window on the composition of the sea. Fermilab experiment E772 has compared yields from the isoscalar targets ${ }^{2} \mathrm{H}$ and $\mathrm{C}$ with yields from a neutron-rich target, W. According to Eq. (2), the ratio of yields per nucleon [8] is $R_{W}(x) \approx 1+0.195(\bar{d}(x)-\bar{u}(x)) /(\bar{d}(x)+\bar{u}(x))$. Because the measured ratio shown in Figure — is consistent with unity, the authors of Ref. [3] concluded that there is no evidence for a large flavor-asymmetry in the light-quark sea of the nucleon. There is, however, no conflict between the dilepton results and the Gottfriedsum-rule defect observed by NMC.

The chiral field theory calculation reviewed above leads with no readjustment of parameters to the thick solid curve [9] shown in Figure [1. That prediction is entirely consistent with the E772 data. So, too, is the flavor-asymmetric fit ("D0") made to deeply inelastic lepton scattering data by Martin, Roberts, and Stirling [10]. The effect on $R_{W}$ is small because the ratio of $\bar{u}(x) / \bar{d}(x)$ is everywhere close to unity for both the chiral quark model and the $\operatorname{MRS}(\mathrm{D} 0)$ fit.

The data do discriminate against ad hoc modifications of the EHLQ structure functions considered by Ellis and Stirling [11] and by us [1]. The dashed line in Figure 11 shows the prediction of the modified EHLQ structure functions with $\bar{u}(x) / \bar{d}(x)=(1-x)^{5.4}$ that we examined in Ref. [1]. This form magnifies the effect of a flavor asymmetry upon $R_{W}$ at large values of $x$, whereas the Gottfried integral is determined by the magnitude of the $(\bar{d}-\bar{u})$ excess, which is determined at small $x$. Even in the interval $0.04<x<0.15$, however, the ad hoc form predicts a larger asymmetry than is observed.

Ellis and Stirling [11] proposed to examine the shape near $x_{F}=0$ of the differential cross section $m^{3} d \sigma / d x_{F} d m$ for the reaction $p d \rightarrow \mu^{+} \mu^{-}+$anything as a probe of the difference between the proton and neutron sea distributions. We show in Figure 2 the Drell-Yan cross section predicted using the structure functions obtained from the chiral quark model. The 
lowest-order calculation has been normalized to the large- $x_{F}$ data using a $K$-factor of 1.4 . It gives an excellent fit to the E772 data, without the suppression of the $x_{F}<0$ cross section given by the ad hoc parton distributions of Ellis and Stirling (cf. Figure 2 of Ref. [3]).

Direct comparison of the yield of dileptons from hydrogen and deuterium targets maximizes the sensitivity of the ratio $R_{A}$ to a flavor asymmetry, because $R_{p} \approx 1-(\bar{d}(x)-$ $\bar{u}(x)) /(\bar{d}(x)+\bar{u}(x))$. A new experiment has been proposed using the E772 apparatus to make this measurement [12]. The prediction of the chiral quark model, shown as the thick solid curve in Figure 3, is slightly below unity because hydrogen is a (maximally) neutronpoor nucleus. The effect of the flavor asymmetry in the nucleon sea is again small, as it is for the $\operatorname{MRS}(\mathrm{D} 0)$ structure functions plotted as the dotted curve. As expected, the ad hoc modification of the EHLQ structure functions produces a very large effect. Future experiments should be able to discriminate against this extreme possibility. Making the case for or against the flavor-asymmetric sea predicted by the chiral quark model presents a considerable challenge to dilepton experiments.

Dilepton production in hadron-nucleus collisions complements deeply inelastic lepton scattering as a probe of the composition of the light-quark sea of the nucleon. Lepton scattering is the more sensitive probe at small $x$, because the difference $F_{2}^{\mu p}-F_{2}^{\mu n}$ is determined by the difference $\bar{d}(x)-\bar{u}(x)$, whereas the Drell-Yan process has greater sensitivity at large $x$, where the parton densities are small, because it measures the fractional difference $(\bar{d}(x)-\bar{u}(x)) /(\bar{d}(x)+\bar{u}(x))$. We find the chiral quark model mechanism compelling and we look forward to new experimental tests of the small excess of $\bar{d}$ over $\bar{u}$ it implies at low values of $x$.

We thank Keith Ellis for supplying predictions for the MRS(D0) structure functions. Fermilab is operated by Universities Research Association, Inc., under contract DE-AC0276CHO3000 with the United States Department of Energy. This work was supported at LBL by the Director, Office of Energy Research, Office of High Energy and Nuclear Physics, Division of High Energy Physics of the U. S. Department of Energy under Contract DEAC03-76SFO0098. 


\section{REFERENCES}

${ }^{1}$ E. J. Eichten, I. Hinchliffe, and C. Quigg, Phys. Rev. D45, 2269 (1992).

2 P. Amaudruz, et al. (New Muon Collaboration), Phys. Rev. Lett. 66, 2712 (1991). The result is given for $Q^{2}=4(\mathrm{GeV} / c)^{2}$.

3 P. L. McGaughey, et al. (E772 Collaboration), Phys. Rev. Lett. 69, 1726 (1992).

${ }^{4}$ K. Gottfried, Phys. Rev. Lett. 18, 1174 (1967); J. D. Bjorken and E. A. Paschos, Phys. Rev. 185, 1975 (1969); J. Kuti and V. F. Weisskopf, Phys. Rev. D4, 3418 (1971).

${ }^{5}$ D. A. Ross and C. T. Sachrajda, Nucl. Phys. B149, 497 (1979); C. López and F. J. Ynduráin, Nucl. Phys. B183, 157 (1981).

${ }^{6}$ A. Manohar and H. Georgi, Nucl. Phys. B234, 189 (1984).

7 E. J. Eichten, I. Hinchliffe, K. Lane, and C. Quigg, Rev. Mod. Phys. 56, 579 (1984), 58, 1065E (1986).

${ }^{8}$ We thank Joel Moss for confirming that the coefficient 0.183 (instead of 0.195) in Ref. [3] is a misprint.

${ }^{9}$ We have evolved the chiral quark model distributions to values of $Q^{2}$ corresponding to the E772 data points and evaluated the Drell-Yan cross section. The thin solid line results from neglecting the effects of $Q^{2}$-evolution and of $d \bar{d}$ annihilations. Those effects are seen to be are small.

10 A. D. Martin, W. J. Stirling, and R. G. Roberts, "New Information on Parton Distributions," Preprint RAL-92-021 DTP/92/16 (unpublished).

11 S. D. Ellis and W. J. Stirling, Phys. Lett. B256, 258 (1991).

12 G. T. Garvey, et al., "Measurement of $\bar{d}(x) / \bar{u}(x)$ in the Proton," Fermilab proposal P866 (unpublished). 


\section{FIGURES}

FIG. 1. The ratio $R_{W} \equiv \sigma_{W} / \sigma_{\text {isoscalar }}$ of dilepton yields per nucleon from tungsten and isoscalar targets as a function of $x_{\text {target }}$. The data are from Fermilab experiment E772, Ref. [3]. Open circles at small $x$ are the ratio before correction for nuclear shadowing. The thick solid curve is our prediction based on chiral field theory, using the full Drell-Yan cross section with parton distributions evaluated at the dimuon mass. The thin solid curve is calculated using the parton distributions from chiral field theory at fixed $Q^{2}=5(\mathrm{GeV} / c)^{2}$ in Eq. (2). The dotted curve shows the prediction of the MRS(D0) parton distributions [10], using Eq. (2). An ad hoc modification of the EHLQ Set 1 structure functions (Ref. [7]) yields the dashed curve (Drell-Yan cross section, parton distributions evolved to $Q^{2}=m^{2}$ ) and the dot-dashed curve (Eq. (2), fixed $\left.Q^{2}=5(\mathrm{GeV} / c)^{2}\right)$.

FIG. 2. Differential cross section $m^{3} d \sigma / d x_{F} d m$ as a function of $x_{F}$ for the reaction $p d \rightarrow \mu^{+} \mu^{-}+$anything at $800 \mathrm{GeV}$ from Fermilab experiment E772, Ref. [3]. The solid curve is our prediction based on chiral field theory for a dimuon mass $m=8.15 \mathrm{GeV} / c^{2}$.

FIG. 3. The ratio $R_{p} \equiv \sigma_{p} / \sigma_{d}$ of dilepton yields per nucleon from hydrogen and deuterium targets as a function of $x_{\text {target }}$. The thick solid curve is our prediction based on chiral field theory, using the full Drell-Yan cross section with parton distributions evaluated at the dimuon mass. The thin solid curve is calculated using the parton distributions from chiral field theory at fixed $Q^{2}=5(\mathrm{GeV} / c)^{2}$ in Eq. (2). The dotted curve shows the prediction of the MRS(D0) parton distributions [10], using Eq. (2). An ad hoc modification of the EHLQ Set 1 structure functions (Ref. [7]) yields the dashed curve (Drell-Yan cross section, parton distributions evolved to $Q^{2}=m^{2}$ ) and the dot-dashed curve (Eq. (2), fixed $\left.Q^{2}=5(\mathrm{GeV} / \mathrm{c})^{2}\right)$. 